\title{
Utility of serum ischemia modified albumin in the early diagnosis of acute coronary syndrome
}

\section{Uday Narendrabhai Vachhani, Janki P. Desai ${ }^{1}$, Chandrakant P. Kamariya, Jignesh H. Gorasia, Kiran P. Chauhan ${ }^{2}$ \\ Department of Biochemistry, Pandit Deendayal Upadhyay Medical College, Rajkot, 'Department of Biochemistry, GMERS Medical College, \\ Ahmedabad, ${ }^{2}$ Department of Biochemistry, GMERS Medical College, Gandhinagar, Gujarat, India}

Address for the Correspondence: Dr. Uday Narendrabhai Vachhani, Department of Biochemistry, Room No. 212, 2nd Floor, Pandit Deendayal Upadhyay Medical College, Jamnagar Road, Rajkot - 360 001, Gujarat, India. E-mail: uday.vachhani@gmail.com

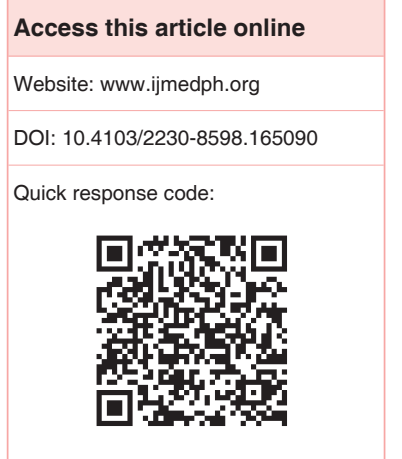

Context: Coronary artery disease (CAD) is the leading cause of death. Early detection of myocardial ischemia can decrease morbidity and mortality due to CAD. Ischemia modified albumin (IMA) is a newer biomarker for early detection of myocardial ischemia in patients of acute coronary syndrome (ACS) as compared to cardiac troponin $\mathrm{T}$ (cTnT). Aims: The aim of this study was to test the utility of "IMA," a new biomarker of myocardial ischemia, in the early diagnosis of ACS. Settings and Design: The cross-sectional study group consisted of 101 patients between the age group of 27 and 85 years having ACS and 100 control from a healthy population. Materials and Methods: Blood was collected from all the enrolled patients immediately after admission, and samples were analyzed for cTnT on the fully automated analyzer and IMA on a spectrophotometer. Statistical Analysis Used: Statistical analysis was carried out by calculation of the area under a curve using receiver operating characteristics (ROC) curve analysis for the IMA test in the 101 patients included in the study population. Results: ROC curve area for IMA was 0.89 (95\% confidence interval 0.81-0.94). At the cut-off $95 \mathrm{U} / \mathrm{ml}$, sensitivity, and specificity were $85 \%$ and $82 \%$ and positive predictive value and negative predictive value (NPV) were $88 \%$ and $78 \%$, respectively. Conclusions: IMA is a useful ischemic marker for diagnosis of myocardial ischemia due to high sensitivity, and it also facilitates to rule out myocardial ischemia due to high NPV.

Key words: Cardiac troponin T, coronary artery disease, myocardial ischemia

\section{INTRODUCTION}

Acute coronary syndromes (ACSs) is a spectrum of disease including unstable angina, associated with reversible myocardial cell injury, Q-wave and non-Q-wave acute myocardial infarction (AMI), non-ST-segment elevation MI and ST-segment elevation MI with large areas of necrosis. ${ }^{[1]}$ Myocardial ischemia occurs either due to a lack of blood flow resulting in a shortage of oxygen and accumulation of waste products in the ischemic tissue. This results in a number of biochemical changes in the cells and if continued, leads to irreversible damage and necrosis. ${ }^{[2]}$ ACS is becoming more common in the developing world such that in India, cardiovascular disease is the leading cause of death in India. ${ }^{[3]}$

Every patient with acute chest pain presenting to Emergency Department should be identified and confirmed for ACS for accurate medical management. ${ }^{[4]}$ Electrocardiography (ECG), troponin and creatine kinase-MB are conventional markers for diagnosis of ACS. These blood markers are extremely sensitive and specific for the identification of patients with myocardial necrosis, but their ability to identify patients with myocardial ischemia remains limited. ECG is rarely abnormal following transient myocardial ischemia, and the ECG at presentation is normal in approximately half of the patients with ACSs. ${ }^{[5]}$ These highly specific markers might give negative results on admission but give positive results hours later because myocardial necrosis is time dependent. Thus, markers able to identify patients with myocardial ischemia without infarction might serve an important role in the clinical setting. ${ }^{[6]}$ Only about $22 \%$ patients develop AMI out of total admission to the cardiac care center. ${ }^{[7]}$

Ischemia modified albumin (IMA) is a newer marker for the detection of myocardial ischemia, in which N-terminal amino acids of human serum albumin are modified by ischemia. The amino 
terminal end (N-terminal) of the albumin molecule is a binding site for transitional metals such as cobalt, copper, and nickel. ${ }^{[8]}$ In the presence of ischemia, the binding capacity of $\mathrm{N}$-terminal of human serum albumin decreases toward cobalt. The principle of this test is to measure unbound cobalt by colorimetric assay. An elevated concentration of IMA has, therefore, been anticipated as a marker of myocardial ischemic injury. This assay is reported to be positive within minutes of ischemia and remains so for up to several hours later, allowing detection before the development of myocardial necrosis. ${ }^{[9,10]}$

\section{MATERIALS AND METHODS}

The study was undertaken from November 2009 to October 2010 after prior approval from hospital Ethical Committee. The cross-sectional study group consisted of 101 patients between the age group of 27 and 85 years, admitted in emergency department and in intensive cardiac care unit within $6 \mathrm{~h}$ of clinical signs and symptoms of ACS. The control group included 100 apparently healthy individuals (age range, 30-70 years) to determine the $95^{\text {th }}$ percentile of a control reference population for the IMA test.

Patients had been diagnosed as ACS was depending upon signs and symptoms as well as ECG changes and status of cardiac troponin $\mathrm{T}$ (cTnT). The diagnosis of ACS was based on criteria defined by the Joint European Society of Cardiology/American College of Cardiology Committee. ${ }^{[11]}$

A cross-sectional study was planned to select serum samples from two distinct patient populations: Group 1 (noncardiac chest pain): Group 1 included 39 out of 101 patients with acute chest pain but no subsequent evidence of myocardial ischemia, labeled as "nonischemic chest pain." Group 2 (cardiac chest pain): Group 2 included 62 patients out of 101 patients with acute chest pain and evidence of early myocardial ischemia, including unstable angina and AMI. These were labeled as "ischemic chest pain."

All the patients with renal diseases, liver cirrhosis, acute stroke, and lung cancer were excluded from this study.

Five milliliter of blood was collected from all the enrolled patients immediately after admission before any treatment was started, in a plain tube for estimation of cTnT and IMA. cTnT were estimated immediately after the separation of sera and then these tests sera were frozen at $-20^{\circ} \mathrm{C}$ for IMA estimation at a later stage. Frozen samples were mixed thoroughly after thawing and re-centrifuged before analysis. Repeat freeze-thaw cycles were avoided to reduce deterioration of samples. The IMA testing was done in batches. All the enrolled patients had an ECG within $1 \mathrm{~h}$ of admission. ECG criteria used to support a clinical diagnosis of myocardial ischemia were ST segment elevation or depression of $1 \mathrm{~mm}$ or more or $\mathrm{T}$ wave inversion $2 \mathrm{~mm}$ or more in 2 contiguous leads.

\section{Albumin cobalt binding (ischemia modified albumin) test}

The colorimetric assay for ischemia modified serum albumin developed by Bar-Or et al. ${ }^{[8]}$ measures free cobalt in serum, after cobalt albumin binding has, takes place. N-terminal of albumin is modified by ischemia and decrease affinity toward cobalt, thus amount of free cobalt is an increase in the reaction mixture. The amount of cobalt bound-albumin and the intensity of the color formation are in an inverse relationship. The method of albumin cobalt binding assay (IMA) involved the addition of $200 \mu \mathrm{l}$ of patient serum to $50 \mu \mathrm{l}$ of a solution of $1 \mathrm{~g} / \mathrm{L}$ cobalt chloride, followed by gentle mixing, and incubated the solution for $10 \mathrm{~min} .50 \mu \mathrm{l}$ dithiothreitol (1.5 g/L solution) was then added, mixed and further incubated for $2 \mathrm{~min}$. Then, $1.0 \mathrm{ml}$ of phenobarbitone buffer $(\mathrm{pH}-$ 8.6) was added. The absorbance of assay mixtures was read at 470 $\mathrm{nm}$ with a ultraviolet/visible T60 UV/VIS Spectrophotometer (PG Instruments Limited, London). The blank was prepared similarly by serum and cobalt chloride without of dithiothreitol and absorbance was read at $470 \mathrm{~nm}$ on the spectrophotometer.

Albumin cobalt binding assay was standardized in the Department of Biochemistry by using different concentration of $\mathrm{CoCl}_{2}$ ranging from 6 to $60.0 \mu \mathrm{g} \mathrm{CoCl} / \mathrm{ml}$. A standard curve was prepared in the range 6-60.0 $\mu \mathrm{g} \mathrm{CoCl} / \mathrm{ml}$. One IMA unit was defined as " $\mu \mathrm{g}$ of free $\mathrm{Co}^{+2}$ in the reaction mixture per $\mathrm{ml}$ of serum sample."'[12] Calibrations were done before running each batch of samples, using freshly prepared $\mathrm{CoCl}_{2}$ and dithiothreitol reagents. Before running each batch control was done using known patient sample from a previous batch, which had been frozen after the test. Imprecision statistics was determined from 20 replicate assays of an unknown sample. The coefficient of variation (CV\%) was calculated using mean values and standard deviation (SD) of 20 replicates. CV\% was $7.8 \%$ in this study.

\section{Cardiac troponin $\mathrm{T}$ assay}

Cardiac troponin $\mathrm{T}$ was measured quantitatively using a electrochemiluminescence immune assay based on electrochemiluminescence technology (fourth generation c'Tn'T, Elecsys 2010, Roche, Mannheim, Germany). ${ }^{[13]}$ The lower detection limit of this assay is $0.01 \mathrm{ng} / \mathrm{ml}$ with a recommended diagnostic threshold of $0.03 \mathrm{ng} / \mathrm{ml}$. Troponin T concentration $\geq 0.03 \mathrm{ng} / \mathrm{ml}$ was considered a positive result.

Receiver operating characteristics (ROC) curve was analyzed and calculation of the area under the curve was done for the IMA test in the 101 patients, included in the study population according to the method of Hanley and McNeil by using MedCalc statistical software Version 12.2.1.0 (MedCalc Software Mariakerke, Belgium). The optimum cut-off for the IMA test was selected from the ROC curve analysis to maximize sensitivity and specificity in the study population. The upper $95^{\text {th }}$ percentile ACB Test value for apparently healthy individuals was calculated using parametric statistics. 


\section{RESULTS}

A total of 101 patients admitted to Emergency Department were selected for the study in the age group of 27-85 years. Of these patients, $71(70 \%)$ were male and $30(30 \%)$ were female. The IMA values for the control reference population $(n=100)$ were between 60.3 and $104.5 \mathrm{U} / \mathrm{ml}$ (mean, $76.2 \mathrm{U} / \mathrm{ml}$; median, $75.8 \mathrm{U} / \mathrm{ml}$ ). The upper $95^{\text {th }}$ percentile was $92.4 \mathrm{U} / \mathrm{ml}$. When serum IMA levels in Group 2 (ischemic chest pain) were compared with Group 1 (nonischemic chest pain), it was found that serum IMA of Group 2 was between 68.9 and $137.2 \mathrm{U} / \mathrm{ml}$ (95\% confidence interval [CI] 76-85 U/ml) with mean $\pm \mathrm{SD}$ as $106.4 \pm 13.3 \mathrm{U} / \mathrm{ml}$. Whereas in Group 1 serum IMA level were between 53.7 and $109.6 \mathrm{U} / \mathrm{ml}(95 \%$ CI $103-110 \mathrm{U} / \mathrm{ml}$ ) with mean $\pm \mathrm{SD}$ as $80.7 \pm 13.9 \mathrm{U} / \mathrm{ml}$. The IMA levels in patients of chest pain with ischemia were significantly higher than patients of chest pain without ischemia and control population $(P<0.0001)$. In the Group 2, $54(87 \%)$ samples were found to have increased IMA levels as compared to $32(82 \%)$ samples in the Group 1 have normal IMA level, shown as a scattered distribution plot between Group 1 and Group 2 [Figure 1]. In Group 2, 26 out of 62 patients were troponin $T$ negative at presentation, but actually they were troponin T positive when the test was repeated after $6 \mathrm{~h}$.

Diagnostic values of IMA, ECG and c'Tn'T together and alone are given in Table 1. When ROC curve was used to evaluate the value of IMA, the area under curve was 0.89 (95\% CI 0.81-0.94) [Figure 2]. At the cut-off $95 \mathrm{U} / \mathrm{ml}$, founded by ROC curve showed the maximum sensitivity of $85 \%$ and specificity of $82 \%$. Positive predictive value (PPV) and negative predictive value (NPV) were $88 \%$ and $78 \%$, respectively. When c'Tn'T and ECG used alone showed the sensitivity of only $58 \%$ and $69 \%$, respectively. Sensitivity and NPV of IMA, cTnT and ECG, when used in combination were $91 \%$. Hence, IMA was able to diagnose more ischemic patient than ECG or c'Tn'T. When IMA, c'Tn'T and ECG used together for diagnosis for ACS, ROC curve analysis showed area under curve as 0.92 (95\% CI $0.83-0.97$ ), sensitivity and NPV of $91 \%$, while specificity and PPV of $82 \%$ respectively [Figure 3]. These three biomarkers demonstrated the significantly greater value of sensitivity and NPV than individual parameters $(P<0.0001)$.

\section{DISCUSSION}

This study illustrated that IMA value was significantly higher in patients with ischemia chest pain as compared to control group

\begin{tabular}{|c|c|c|c|c|}
\hline Test & $\begin{array}{c}\text { Sensitivity } \\
(\%)\end{array}$ & $\begin{array}{c}\text { Specificity } \\
(\%)\end{array}$ & $\begin{array}{l}\text { PPV } \\
\text { (\%) }\end{array}$ & $\begin{array}{l}\text { NPV } \\
(\%)\end{array}$ \\
\hline IMA (cut-off $95 \mathrm{U} / \mathrm{ml}$ ) & 86 & 82 & 88 & 78 \\
\hline $\operatorname{cTnT}(\geq 0.03)$ & 58 & 97 & 97 & 59 \\
\hline ECG & 69 & 95 & 95 & 66 \\
\hline IMA+troponin+ECG & 91 & 82 & 81 & 91 \\
\hline
\end{tabular}

IMA = Ischemia modified albumin, cTnT = Cardiac troponin T,

$\mathrm{ECG}=$ Electrocardiography, $\mathrm{ACS}=$ Acute coronary syndrome, NPV = Negative predictive value, $\mathrm{PPV}=$ Positive predictive value and nonischemic chest pain group. In this study, cut-off value of IMA $95 \mathrm{U} / \mathrm{ml}$, derived by ROC curve was relatively higher than $85 \mathrm{U} / \mathrm{ml}$, because IMA was performed by manual method on

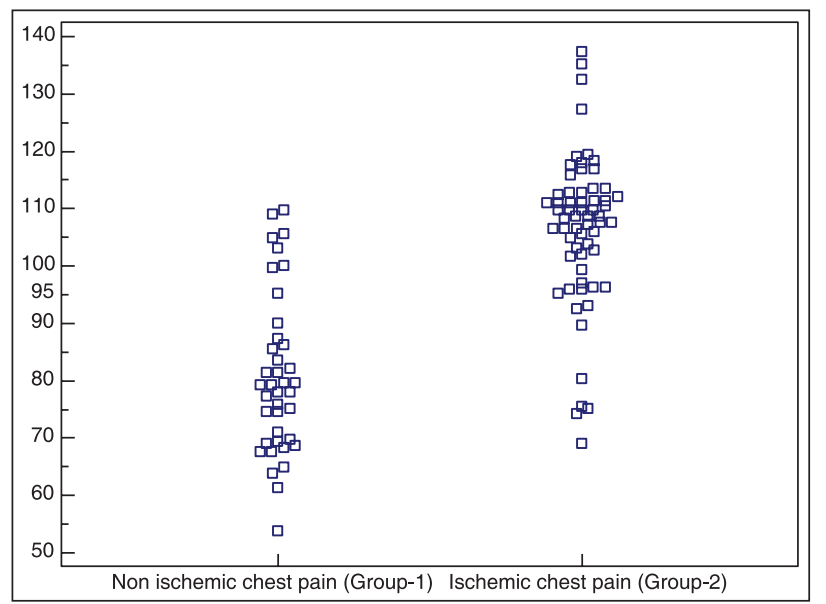

Figure 1: Distribution of ischemia modified albumin value amongst patients of chest pain at presentation

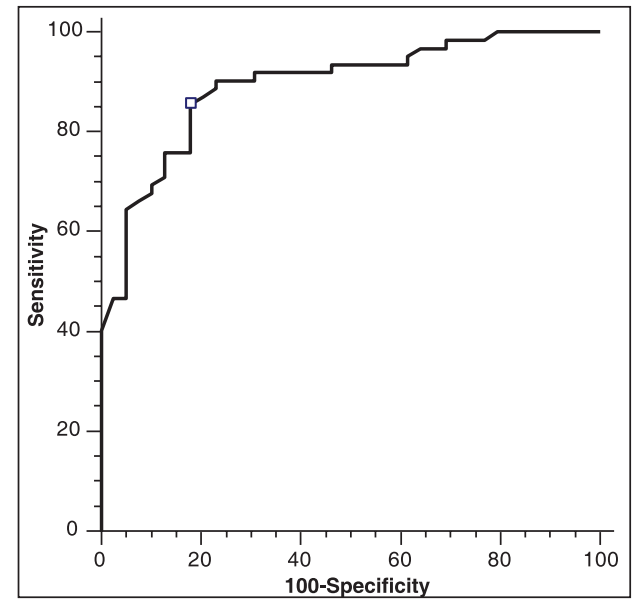

Figure 2: Receiver operating characteristics curve for serum ischemia modified albumin level amongst ischemic and nonischemic patients

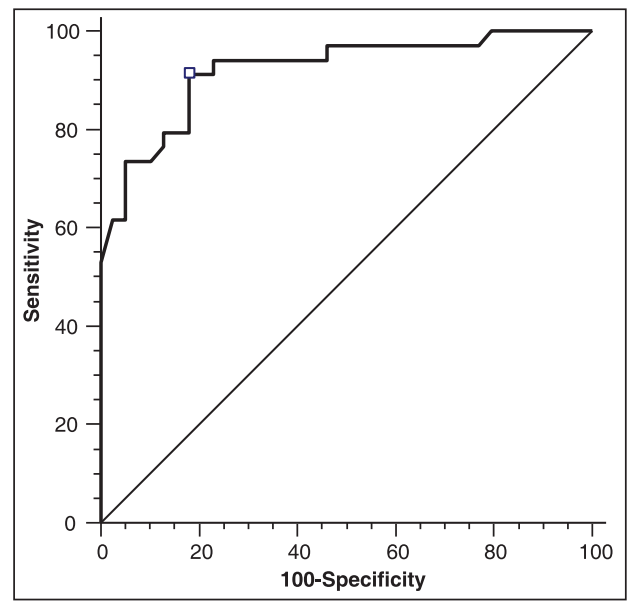

Figure 3: Receiver operating characteristics curve for serum ischemia modified albumin, cardiac troponin $\mathrm{T}$ and electrocardiography amongst ischemic and nonischemic patients 
spectrophotometer, while it was performed on fully automated chemistry analyzer in previous studies done by Sinha et al. ${ }^{[14]}$ and Collinson et al. ${ }^{[15]}$

ROC curve analysis of IMA showed higher sensitivity (86\%) and NPV (78\%) than cTnT and ECG at cut-off IMA value of $95.1 \mathrm{U} / \mathrm{ml}$ were similar to the result of Sinha et al. ${ }^{[14]}$ and Takhshid et al. ${ }^{[16]}$ studies. If we decrease IMA cut-off value to $87 \mathrm{U} / \mathrm{ml}$, it gives higher sensitivity (92\%) and NPV (84\%), but it will decrease specificity to $69 \%$. In this study cut-off for reference population is $92.4 \mathrm{U} / \mathrm{ml}$ ( $95^{\text {th }}$ percentile), but use of a cut-off of $95 \mathrm{U} / \mathrm{ml}$ was to minimize the number of false positive and false negative results. This was the reason for taking cut off $95 \mathrm{U} / \mathrm{ml}$ for this study.

Receiver operating characteristics curve analysis for IMA, cTnT and ECG together showed sensitivity and NPV of 91\%, which was significantly greater than that of shown by IMA, c'Tn'T and ECG alone. These findings were nearly similar to result of Sinha et al..$^{[14]}$ and Takhshid et al. ${ }^{[16]}$ studies. Our study proved that IMA had high sensitivity for early diagnosis of ACS and high negative predictive to rule out cardiac ischemia in nonischemic chest pain patients, but the specificity of IMA was lower than $\mathrm{c}^{\top} \mathrm{Tn}$ T $\mathrm{T}$ in patients at presentation, and therefore IMA, can be used as diagnostic marker along with c'TnT and ECG in ACS. If value of IMA and c'TnT are $<95 \mathrm{U} / \mathrm{ml}$ and $<0.03$ respectively with nondiagnostic ECG, NPV was $91 \%$ for ACS in this study. IMA, cTnT and ECG comprise an excellent combination for noninvasive tests. When the physician is trying to make a decision whether to send the patient home or keep him in the hospital, the information from these three tests in combination is very useful.

Limitation of this study was IMA test returns to baseline within 8-12 h after an ischemic event and many of the patients were excluded from this study due to late presentation.

\section{CONCLUSION}

From this study, it can be concluded that albumin cobalt binding test for IMA is easy and simple end point method that can be measured by spectrophotometer and can be easily established in a small scale laboratory. This study suggests that patients presenting with acute chest pain and have high IMA values should be assessed and followed up carefully, even if cardiac troponin and ECG are negative. When IMA, cardiac troponin, and ECG used in combination, clinicians can easily make a diagnosis of myocardial ischemia in patients presenting to Emergency Department. These three biomarkers could allow early discharge of nonischemic chest pain patients more safely and cost effectively, due to higher NPV of these biomarkers.

\section{REFERENCES}

1. Christenson RH, Duh SH, Sanhai WR, Wu AH, Holtman V, Painter P, et al. Characteristics of an Albumin Cobalt Binding Test for assessment of acute coronary syndrome patients: A multicenter study. Clin Chem 2001;47:464-70.

2. Thygesen K, Alpert JS, White HD, Joint ESC/ACCF/AHA/WHF Task Force for the Redefinition of Myocardial Infarction, Jaffe AS, Apple FS, et al. Universal definition of myocardial infarction. Circulation 2007;116:2634-53.

3. Mukherjee AK. Prediction of coronary heart disease using risk factor categories. J Indian Med Assoc 1995;93:312-5.

4. Storrow AB, Gibler WB. Chest pain centers: Diagnosis of acute coronary syndromes. Ann Emerg Med 2000;35:449-61.

5. Singer AJ, Brogan GX, Valentine SM, McCuskey C, Khan S, Hollander JE. Effect of duration from symptom onset on the negative predictive value of a normal ECG for exclusion of acute myocardial infarction. Ann Emerg Med 1997;29:575-9.

6. Anwaruddin S, Januzzi JL Jr, Baggish AL, Lewandrowski EL, Lewandrowski KB. Ischemia-modified albumin improves the usefulness of standard cardiac biomarkers for the diagnosis of myocardial ischemia in the emergency department setting. Am J Clin Pathol 2005;123:140-5.

7. Graff LG, Dallara J, Ross MA, Joseph AJ, Itzcovitz J, Andelman RP, et al. Impact on the care of the emergency department chest pain patient from the chest pain evaluation registry (CHEPER) study. Am J Cardiol 1997;80:563-8.

8. Bar-Or D, Lau E, Winkler JV. A novel assay for cobalt-albumin binding and its potential as a marker for myocardial ischemia-a preliminary report. J Emerg Med 2000;19:311-5.

9. Bar-Or D, Lau E, Rao N, Bampos N, Winkler JV, Curtis CG. Reduction in the cobalt binding capacity of human albumin with myocardial ischemia. Ann Emerg Med 1999;34:S56.

10. Bar-Or D, Winkler JV, Vanbenthuysen K, Harris L, Lau E, Hetzel FW. Reduced albumin-cobalt binding with transient myocardial ischemia after elective percutaneous transluminal coronary angioplasty: A preliminary comparison to creatine kinase-MB, myoglobin, and troponin I. Am Heart J 2001;141:985-91.

11. The Joint European Society of Cardiology/American College of Cardiology Committee. Myocardial infarction redefined-a consensus document of the Joint European Society of Cardiology/American College of Cardiology Committee for the Redefinition of Myocardial Infarction. J Am Coll Cardiol 2000;36:959-9.

12. Chawla R, Goyal N, Calton R, Goyal S. Ischemia modified albumin: A novel marker for acute coronary syndrome. Indian J Clin Biochem 2006;21:77-82.

13. Roche Diagnostics. Troponin T STAT (Short Turn Around Time), CARDIAC T 04660307190 2007-07. 2007.

14. Sinha MK, Roy D, Gaze DC, Collinson PO, Kaski JC. Role of "Ischemia modified albumin", a new biochemical marker of myocardial ischaemia, in the early diagnosis of acute coronary syndromes. Emerg Med J 2004;21:29-34.

15. Collinson PO, Gaze DC, Bainbridge K, Morris F, Morris B, Price A, et al. Utility of admission cardiac troponin and "Ischemia Modified Albumin" measurements for rapid evaluation and rule out of suspected acute myocardial infarction in the emergency department. Emerg Med J 2006;23:256-61.

16. Takhshid MA, Kojuri J, Tabei SM, Tavasouli AR, Heidary S, Tabandeh M. Early diagnosis of acute coronary syndrome with sensitive troponin I and ischemia modified albumin. Iran Cardiovasc Res J 2010;4:144-1.

How to cite this article: Vachhani UN, Desai JP, Kamariya CP, Gorasia JH, Chauhan KP. Utility of serum ischemia modified albumin in the early diagnosis of acute coronary syndrome. Int $\mathrm{J}$ Med Public Health 2015;5:340-3.

Source of Support: Nil, Conflicts of Interest: None declared. 